Classification

Physics Abstracts

$74.70-71.55 \mathrm{~J}$

\title{
Superconductivity, localization and crystallographic phase transition in $\mathrm{La}_{2} \mathrm{Sr}_{x} \mathrm{CuO}_{4-y}$
}

\author{
W. Kang, G. Collin, M. Ribault, J. Friedel, D. Jérôme, J.M. Bassat ${ }^{+}$, J.P. Coutures ${ }^{+}$and $^{+}$ \\ Ph. Odier ${ }^{+}$ \\ Laboratoire de Physique des Solides, Université Paris-Sud, 91405 Orsay, France
}

${ }^{+}$CRPHT-CNRS, 45071 Orléans Cedex 2, France

(Reçu le 11 mai 1987)

\begin{abstract}
Résumé.- Nous présentons une étude par résistivité de la supraconduction de la série $\mathrm{La}_{2-x} \mathrm{Sr}_{x} \mathrm{CuO}_{4-y}$ à l'aide d'échantillons cractérisés par analyse thermogravimétrique en ce qui concerne la teneur en oxygène. On observe la supraconductivité d'une part pour des valeurs de $x$ non nulles avec un maximum de $T_{\mathrm{c}}$ au voisinage de la transition de phases tetragonal-orthorombique et d'autre part dans le composé nonsubstitué $\mathrm{La}_{2} \mathrm{Cu} \mathrm{O}_{4-y}$. Une localisation importante des électrons est observée lorsque $x \approx 0,04$. Ces résultats sont en accord avec un modèle BCS simple pour lequel le remplissage de bande est déterminé par le dopage au strontium. L'existence de localisation pourrait provenir de la compétition entre les effets des lacunes d'oxygène et du dopage au strontium. Lorsque $x \approx 0,04$ la situation de bande demi-remplie permet les diffusions de type Umklapp. Nos résultats suggèrent une contribution des fluctuations antiferromagnétiques au mécanisme de "pairing" supraconducteur.
\end{abstract}

\begin{abstract}
We report a resistive investigation of superconductivity in the system $\mathrm{La}_{2-x} \mathrm{Sr}_{x} \mathrm{CuO}_{4-y}$ on samples which have been analysed by thermogravimetric techniques for oxygen deficiency determination. A maximum superconductive transition temperature is observed in $\mathrm{La}_{2-x} \mathrm{Sr}_{x} \mathrm{CuO}_{4-y}$ near the tetragonal-orthorhombic phase transition, together with a resurgence of high temperature superconductivity in non-substituted $\mathrm{La}_{2} \mathrm{CuO}_{4-y}$. Drastic localization effects are observed in the vicinity of $x=0.04$ with no sign of superconductivity at low temperature. These observations are coherent with a simple BCS scheme with a band filling dominated by Sr-doping and localization effects dominated by an interplay between 0 vacancies and Sr-doping. This situation leads to half-filling of the band near $x=0.04$ with enhanced electron localization via Umklapp scattering through a near nested Fermi surface. Moreover, our data suggest some contribution of antiferromagnetic fluctuations to the superconducting pairing mechanism.
\end{abstract}

The recent report of superconductivity at a temperature higher than $30 \mathrm{~K}$ in a ternary lanthanumalkaline earth copper oxide by Bednorz and Muller [1] has stimulated a very impressive amount of work and has raised several fundamental experimental and theoretical problems which are a challenge for solid-state chemists and physicists.

The high $T_{\mathrm{c}}$ superconducting phases belong to a class of materials which have heen around for quite some time : the lanthanum copper oxides $\mathrm{La}_{2} \mathrm{CuO}_{4}$ [2]. The structure of $\mathrm{La}_{2} \mathrm{CuO}_{4}$ studied in details by Longo and Raccah [3] shows it derives from the tetragonal structure of the 2D magnetic perovskite $\mathrm{K}_{2} \mathrm{NiF}_{4}$ but also exhibits a small orthorhombic distortion. Later, metallic conductivity was reported in the solid solution $\mathrm{La}_{2-x} \mathrm{M}_{x} \mathrm{CuO}_{4}(\mathrm{M}=$ alkaline earth) [4] and the group of Caen has studied in details the oxygen intercalation in this class of materials and shown they usually are oxygen deficient, namely
$\mathrm{La}_{2-x} \mathrm{M}_{x} \mathrm{CuO}_{4-y}$ [5]. In the present work we deal with materials which are either non-substituted or $\mathrm{Sr}$ substituted. There exists a consensus in the recent literature regarding the optimum amount of $\mathrm{Sr}$ substitution. It is around $x=0.15$ where both $T_{\mathrm{c}}$ is the highest $(\approx 35 \mathrm{~K})$ and the diamagnetic expulsion is the largest $(\approx 50 \%)$.

However, the $\mathrm{La}_{2} \mathrm{CuO}_{4}$ family exhibits a wealth of interesting features which are worth mentioning as they could be related to the occurrence of superconductivity.

(i) A small orthorhombic distortion exists in $\mathrm{La}_{2} \mathrm{CuO}_{4}$ at room temperature and vanishes at a temperature of 533K [3] above which the tetragonal $\mathrm{K}_{2} \mathrm{NiF}_{4}$ structure becomes stable.

(ii) Substitution of Sr for La preserves the distorted orthorhombic structure of lower temperature but increases the average valence charge of the 
copper ions from the charge $\mathrm{Cu}^{2+}$ of $\mathrm{La}_{2} \mathrm{CuO}_{4}$, in order to maintain charge neutrality.

(iii) Oxygen is known to deintercalate and reintercalate easily in $\mathrm{La}_{2-x} \mathrm{Sr}_{x} \mathrm{CuO}_{4-y}(y \geqslant 0)$ even at moderate temperature and pressure, with a strong concomitant influence on transport properties [5].

Also, several experiments [6-9] on these materials have recently proved the dominant role played by the oxygen stoichiometry in the occurrence or disappearence of the superconductivity phenomena. Low oxygen content, i.e. high oxygen vacancy concentration, which promotes a decrease in the net hole concentration, favors carrier localization and possibly suppresses superconductivity phenomena. It has thus been considered as an important point to evaluate quantitatively the net oxygen content of several materials in which superconductivity properties have been studied.

Hence, the main purpose of this work is a study of the superconductivity occurrence in the $\mathrm{La}_{2-x} \mathrm{Sr}_{x} \mathrm{CuO}_{4-y}$ series in relation with $\mathrm{Sr}$ substitution, content of oxygen vacancies and the proximity of the tetragonal to orthorhombic $(T \rightarrow 0)$ distortion.

Our results confirm that divalent alkaline-earth substitution is not a prerequisite for the observation of superconductivity in the $\mathrm{La}_{2} \mathrm{CuO}_{4}$ series [9]. Moreover, a detailed investigation at various substitution levels suggest the possibility of an interplay between the $T \rightarrow 0$ phase transition and superconductivity which is reminiscent of the interplay between superconductivity and charge density wave formation observed in 2D layered dichalcogenides [10].

All oxides studied in this work were prepared in the same way : mixing of freshly calcined lanthanum oxide, copper oxide and strontium carbonate with subsequent firing in air, followed by several grinding and annealing procedures. Final treatment consists in overnight annealing of pressed pellets at $1100^{\circ} \mathrm{C}$ in air and spontaneous cooling after switching off the furnace. X-ray powder patterns performed on Guinier-Laine camera $\left(\mathrm{CuK}_{\alpha}\right)$ indicate that the materials are perfectly single-phased, no trace of impurity can be detected even on overexposed photographs. The materials exhibit at room temperature the orthorhombic distortion up to $x=0.09$ and are strictly tetragonal for higher strontium concentrations. It is the same source of materials which has been used for an X-ray determination of the $T \rightarrow 0$ phase diagram [11].

Small parallelepipedic samples of typical size $\approx 0.5 \times 0.5 \times 6 \mathrm{~mm}$ were cut in the sintered pellets with a diamond saw and used for resistivity measurements with four silver paint contacts. Either low fequency $A C$ or DC currents were used to measure resistivity.

The oxygen content has been determined by the weight loss of the sample under reducing conditions.
A symmetrical micro-balance from SETARAM (1500) has been used between 20 and $1000^{\circ} \mathrm{C}$. The sintered samples were grinded in an agath mortar just before their introduction in the thermobalance. A typical weight of 100 to $200 \mathrm{mg}$ has been used. The sample is then out gassed for an hour under vacuum $\left(10^{-1}\right.$ torr $)$ at room temperature in order to remove part of the adsorbed species. Argon is then introduced at a flow rate of a few litres per hour. The temperature is then raised up to $1000^{\circ} \mathrm{C}$ at a rate of $300^{\circ} \mathrm{C} / \mathrm{h}$. An initial weight loss is observed between 20 and $550^{\circ} \mathrm{C}$, followed by a range of temperatures in which no weight reduction is observed. At $1000^{\circ} \mathrm{C}$ the weight remains constant (under Ar) at least for more than an hour. After that initial step, $\mathrm{Ar}-5 \% \mathrm{H}_{2}$ is introduced in the thermobalance at the same flow rate as previously. The sample is thus reduced rapidly, in $30 \mathrm{~min}$, in its constituants $\mathrm{La}_{2} \mathrm{O}_{3}, \mathrm{SrO}$ and $\mathrm{Cu}$ metal. It was checked that the weight remains stable for more than one hour after complete reduction.

The resistivity data at various substitution levels below $60 \mathrm{~K}$ are presented in figure 1 . All samples for $0.08 \leqslant x \leqslant 0.20$ exhibit superconductivity. The critical temperature in figure $2 \mathrm{a}$ is defined by the temperature corresponding to the mid-point of the resistivity transition. The width $\Delta T$ of the superconducting transition is characterized by the temperature incre-

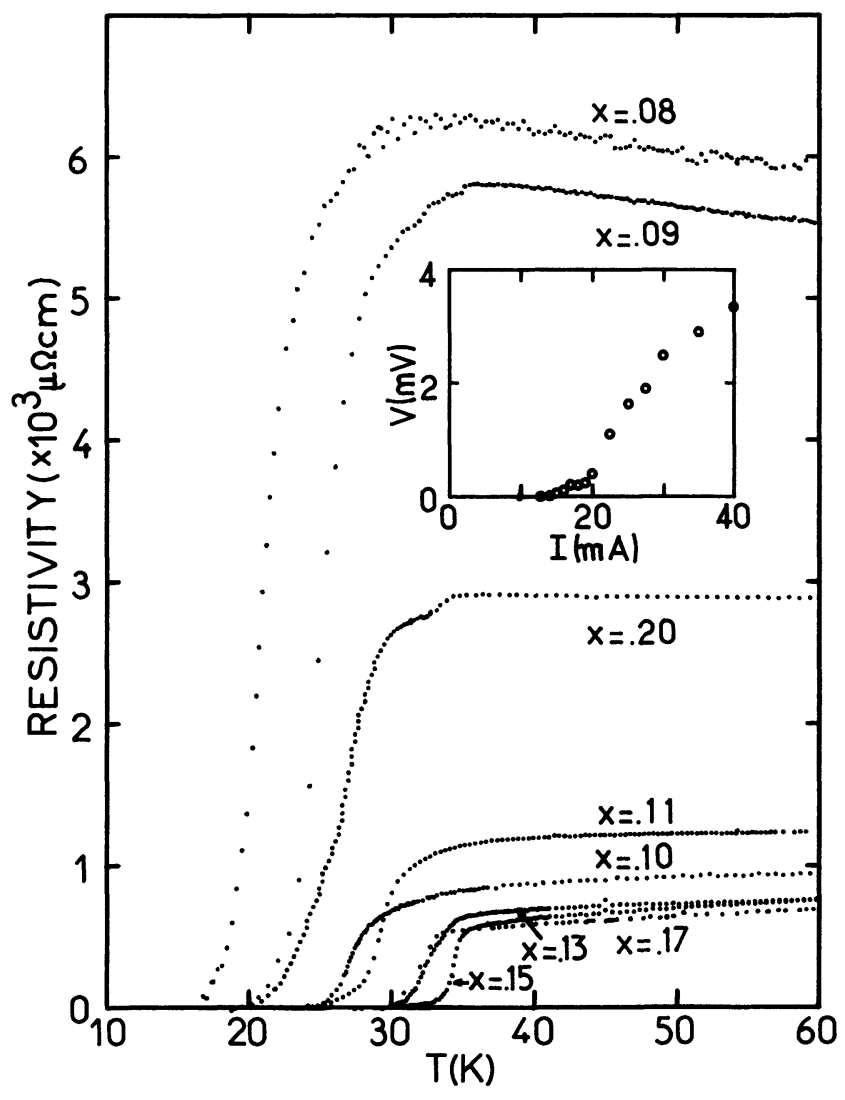

Fig.1.- Temperature dependence of the resistivity versus the Sr-substitution ratio. The insetshows the current-voltage characteristics of the $x=0.15$ sample at $4.2 \mathrm{~K}$. 
ment between $10 \%$ and $90 \%$ of the resistive transition.

Furthermore, we may notice from figure 1 a drastic dependence of $T_{c}$ and of the normal state resistivity on $x$. The value $x=0.15$ provides about the lowest normal state resistivity which goes along with the optimum range for superconductivity. This was also derived from the concentration dependence of the Meissner effect [12] which shows the maximum fraction of ideal diamagnetism (about 50\%) around $x \approx 0.15-0.20$. The inset of figure 1 shows the behaviour of the resistance at $4.2 \mathrm{~K}$ versus $\mathrm{DC}$ current for $x=0.15$. The low value of the critical current may be attributed to the granular character of the material but the critical current is still much larger at $x=0.15$ than in the case of "pure" $\mathrm{La}_{2} \mathrm{CuO}_{4-y}$ which will be discussed later in this article.

Data of $T_{\mathrm{c}}$ and transition width versus $x$ are reported in figure $2 \mathrm{a}$. A maximum of $T_{\mathrm{c}}$ is cleary observed at $x=0.15$. A minimum width at $x=0.15$

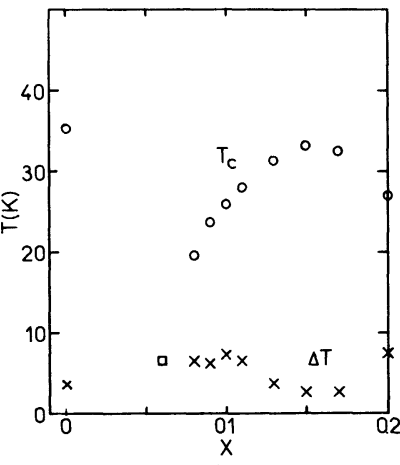

(a)

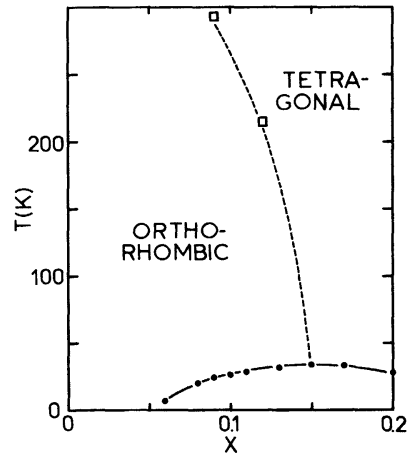

(b)
Fig.2.- a) Critical temperatures and transition widths for various samples, $\square$ denotes the temperature of the anomaly for the $x=0.06$ sample as explained in the text. b) Phase diagram of $\mathrm{La}_{2}{ }_{-x} \mathrm{Sr}_{x} \mathrm{CuO}_{4-y}$.

is expected as $T_{\mathrm{c}}$ is passing through a maximum at the same value of $x$. However, figure 2a unambiguously shows that the drop of $T_{\mathrm{c}}$ observed on either sides of $x=0.15$ is a genuine effect. It cannot be attributed to some kind of smearing of the transition. The $T_{\mathrm{c}}=6.6 \mathrm{~K}(\square)$ point corresponds to a small but sharp drop of the resistivity occurring at this temperature in a $x=0.06$ sample. However, this sample does not show zero resistivity below $T_{\mathrm{c}}$. This anomaly is probably related to superconductivity but it is by no means a bulk phenomenon at $x=0.06$. At the concentration $x=0.04$ the temperature coefficient of the resistance is negative at low temperature with no sign of any superconducting anomaly down to 4.2K (Fig. 3).

Figure $2 \mathrm{~b}$ deals with the interplay between the $T \rightarrow 0$ phase transition and superconductivity. The $x=0.10$ and 0.12 data points have been taken from a recent $\mathrm{X}$-ray study [11]. The dashed line is a guide

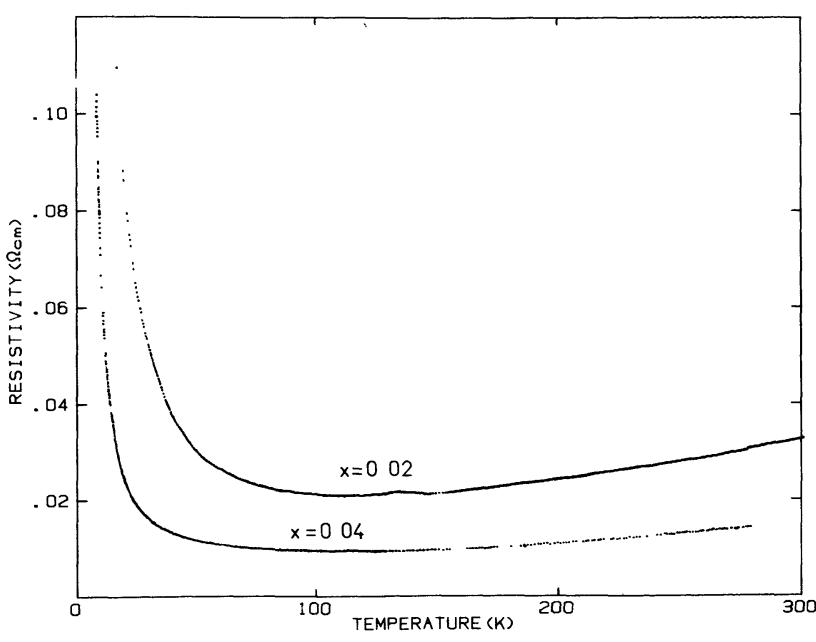

Fig.3.- Resistivity behaviour in $x=0.02$ and $x=0.04$ samples. Localization is evident at low temperature.

for the eye. Since the slope of the crystallographic phase transition line is likely to go to $-\infty$ as the transition temperature approaches zero temperature for thermodynamical reasons we feel confident that figure $2 b$ is a fair representation of the phase diagram in the vicinity of $x=0.15$. Moreover, the diagram of figure $2 b$ is in fair agreement with neutron diffraction studies performed on powder samples [13] which imply that the $T \rightarrow 0$ transition of the $x=0.15$ sample is located above $60 \mathrm{~K}$. It also indicates that the vanishing of the orthorhombic distortion represents the optimum situation for the onset of superconductivity.

The amount of additional holes (electrons) and relative weight losses determined from thermogravimetric studies are given in table I. We notice that the additional hole concentration is always smaller than the amount inferred from the $\mathrm{Sr}$ concentration but increases more or less monotonously with $x$. In the case of $\mathrm{Sr}$-substituted $\mathrm{La}_{2} \mathrm{CuO}_{4}$ the oxygen vacancy concentration is in the range of a few percent. The hole concentration estimated by the charge neutrality is thus in the range of 3 to $9 \times 10^{20} \mathrm{~cm}^{-3}$ from $x=0.08$ to $x=0.15$. These measurements are in general agreement with previous results from Raveau et al. [14]. As far as pure $\mathrm{La}_{2} \mathrm{CuO}_{4}$ is concerned, the sample slowly cooled from $1000^{\circ} \mathrm{C}$ in air gives a small but significant vacancy concentration of about $4 \%$ excess electrons per formula unit. One has thus to account for oxygen vacancies which decrease the hole concentration. Two possibilities can be considered: Lanthanum vacancies coming from structural basis considerations [15] and/or negative charge carrier excess.

As shown in figure $2 \mathrm{a}$, we confirm the existence of a superconducting transition in unsubstituted $\mathrm{La}_{2} \mathrm{CuO}_{4-y}$ which is usually reported as exhibiting a semiconducting behaviour at low temperature 
$[8,16]$. In our sample, the onset of superconductivity in pure $\mathrm{La}_{2} \mathrm{CuO}_{4-y}$ is quite different from what it is in Sr-substituted materials. The resistivity of $\mathrm{La}_{2} \mathrm{CuO}_{4-y}$ is fairly high at room temperature and increases further by a factor about 50 down to the superconducting transition (Fig. 4). Given the sensitivity of our measuring system we can say that a zero resistance is achieved below $T_{\mathrm{c}}$ as long as the current passing through the sample does not exceed about $10 \mu \mathrm{A}$ (see insert of Fig.4). The critical current of $4.2 \mathrm{~K}$ is roughly $10^{3}$ times lower than that of the $x=0.15$ samples.

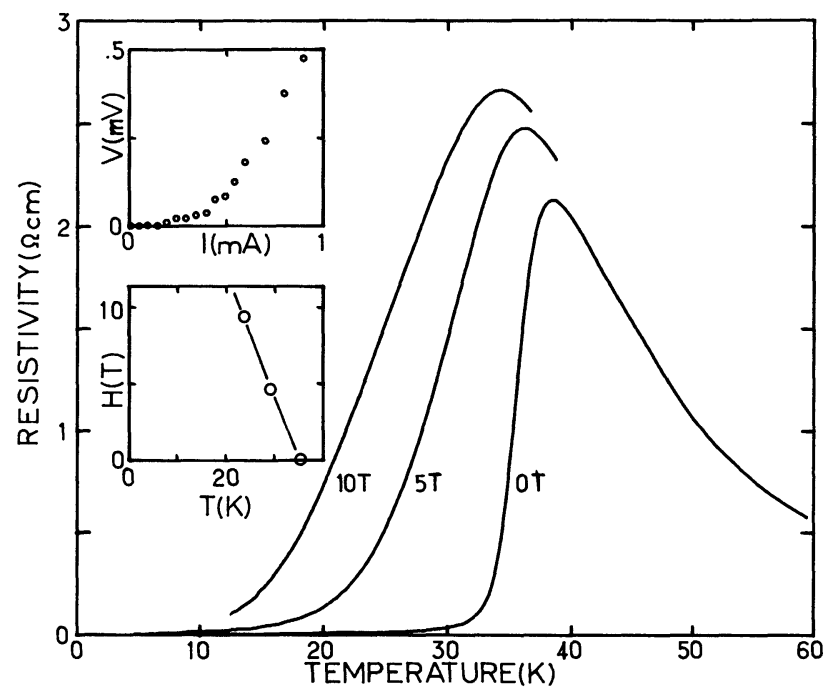

Fig.4.- Temperature dependence of the resistivity of $\mathrm{La}_{2} \mathrm{CuO}_{4-y}$ under three different magnetic fields. The two insets show the current-voltage characteristics at $4.2 \mathrm{~K}$ and the critical field temperature dependence.

Furthermore, the shift of the transition under magnetic field $\left(\mathrm{dH}_{\mathrm{c}_{2}} / \mathrm{d} T \approx 1.12 \mathrm{~T} / \mathrm{K}\right)$ displayed in figure 4 supports the superconducting nature of the resistive anomaly of "pure" $\mathrm{La}_{2} \mathrm{CuO}_{4-y}$. Considering the quite similar values of $T_{\mathrm{c}}$ and $\mathrm{dH}_{\mathrm{c}_{2}} / \mathrm{d} T$ values in pure and Sr-substituted $\mathrm{La}_{2} \mathrm{CuO}_{4-y}$, we may infer that the electronic structure should not be that different in both cases. However, superconductivity in the present $\mathrm{La}_{2} \mathrm{CuO}_{4-y}$ sample does not seem to be a bulk phenomenon. The critical current is extremely low and a preliminary attempt to measure the Meissner effect has failed to detect more than $0.1 \%$ flux expulsion [17]. Also in this sample, we observed a weak resistivity anomaly at around $230 \mathrm{~K}$ which seems to correspond with that from susceptibility measurements $[18,19]$.

Thermogravimetric results reported in table $\mathrm{I}$ do require some comments. The oxygen deficiency is small in all Sr-substituted samples in agreement with the early work of Michel and Raveau [5] and with a neutron diffraction study [16] establishing an upper limit of $1 \%$ for oxygen vacancies in
Table I.- Results from the thermogravimetry. $-\Delta m / m$ measured from $1000{ }^{\circ} \mathrm{C}$. The relative weight loss between 20 and $550^{\circ} \mathrm{C}$ is smaller than $4 \times 10^{-3}$ for the Sr-substituted sample.

$\begin{array}{cccc}x & -\Delta m / m & \begin{array}{c}\text { Estimated } \\ \text { composition }\end{array} & \begin{array}{c}\text { Excess } \\ \text { concentrations of } \\ \text { electrons }(-) \text { or } \\ \text { holes }(+)\end{array} \\ 0.00 \mathrm{~A} & 3.86 \times 10^{-2} & \mathrm{La}_{2} \mathrm{CuO}_{3.98} & -0.04 \\ 0.00 \mathrm{~B} & 3.86 \times 10^{-2} & \mathrm{La}_{2} \mathrm{CuO}_{3.98} & -0.04 \\ 0.09 & 4.06 \times 10^{-2} & \mathrm{La}_{1.91} \mathrm{Sr}_{0.09} \mathrm{CuO}_{3.97} & +0.03 \\ 0.10 & 4.155 \times 10^{-2} & \mathrm{La}_{1.90} \mathrm{Sr}_{0.10} \mathrm{CuO}_{3.99} & +0.08 \\ 0.11 & 4.14 \times 10^{-2} & \mathrm{La}_{1.89} \mathrm{Sr}_{0.11} \mathrm{CuO}_{3.98} & +0.07 \\ 0.15 & 4.20 \times 10^{-2} & \mathrm{La}_{1.85} \mathrm{Sr}_{0.15} \mathrm{CuO}_{3.97} & +0.09\end{array}$

$\mathrm{La}_{1.85} \mathrm{Ba}_{0.15} \mathrm{CuO}_{4-y}$. On the other hand, "pure" $\mathrm{La}_{2} \mathrm{CuO}_{4-y}$ exhibits a non zero content of oxygen vacancies which amounts to a concentration of $4 \%$ additional electrons in samples A and B. Sample A which shows superconducticity was slow cooled in air after firing at $1100^{\circ} \mathrm{C}$ whereas sample B quenched in air exhibits a more insulating character and no superconductivity at low temperature. Quite similarly Cava and van Dover [8] could not find superconductivity in a quenched powder of $\mathrm{La}_{2} \mathrm{CuO}_{4}$.

Taken in isolation, the results reported here are not necessarily contradictory with the various theories of strong coupling superconductivity based on a non conducting pure and stoichiometric $\mathrm{La}_{2} \mathrm{CuO}_{4}$ compound and on superconductivity associated with doping [20-23]: in this light, $\mathrm{La}_{2-x} \mathrm{Sr}_{x} \mathrm{CuO}_{4-y}$ would be superconductive because of $\mathrm{Sr}$ doping ; $\mathrm{La}_{2} \mathrm{CuO}_{4-y}$ because of $\mathrm{O}$ deficiency. However, two points must be stressed: (i) the behaviour of the conductivity above $T_{\mathrm{c}}$, which is very sensitive to $O$ deficiency, does not look like being due to the approach of a metal-insulator phase change by gap opening; (ii) the rather small sensitivity of $T_{c}$ on $\mathrm{O}$ deficiency [9]. They show that simple minded strong coupling scheme should be modified to take such observations into account.

One can also refer to the simple BCS scheme [2426], treating $\mathrm{Sr}$ doping and $\mathrm{O}$ deficiency effects as only changing the band filling in a rigid band model. In such a picture, $T_{\mathrm{c}}$ should increase in the strongly doped tetragonal phase when $x-2 y$ is reduced, because the Fermi level $E_{\mathrm{M}}$ would approach the central van Hove anomaly $E_{0}$ (Fig. 5a). Below a critical value of $(x-2 y)$, the orthorhombic phase should become more stable, with the Fermi level near to but above the lower van Hove anomaly $E_{1}$ (Fig. 5b). Further reduction of $(x-2 y)$ would push the Fermi level away from $E_{1}$ and this could reduce $T_{\mathrm{c}}$. Hence, a maximum of $T_{\mathrm{c}}$ could occur near the boundary of the phase change, as observed experimentally. Analogous interplays between 
superconductivity and phase change are known: cubic to tetragonal phase change in quasi 1D $\mathrm{V}_{3} \mathrm{Si}$ type of compounds with doping [27], suppression of a CDW under pressure in quasi $2 \mathrm{D}$ transition dichalcogenides such as $2 \mathrm{H}-\mathrm{NbSe}_{2}$ or $4 \mathrm{H}_{\mathrm{b}}-\mathrm{TaS}_{2}$ [10]. In all cases, the phase change is associated with a reduction in the density of states near the Fermi level, which lowers $T_{\mathrm{c}}$. In the same rigid band model, the $T_{\mathrm{c}}$ observed in $\mathrm{La}_{2} \mathrm{CuO}_{4-y}$ could be attributed to a raising of the Fermi level near to the second van Hove anomaly $E_{2}$ (Fig. 5b).

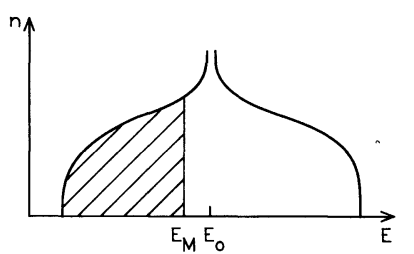

(a)

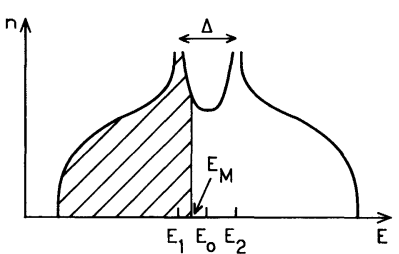

(b)
Fig.5.- Band structure in the simple BCS scheme: a) Tetragonal phase, b) Orthorhombic phase.

We believe that such a simple minded description also requires some modifications.

Because the $T \rightarrow 0$ distortion is small (about $1 \%$ ), the energy splitting of figure $5 \mathrm{~b}$ is modest. As a result, for any position of the Fermi level between the two van Hove anomalies, they should add their effects, leading to a $T_{\mathrm{c}}$ not very sensitive to band filling in this range [28]. To explain the total absence of superconductivity in the vicinity of $x=0.04$, one must invoke the presence, in this range of $\mathrm{Sr}$ concentration and in our samples (with $y \approx 0.02$ ), of some new insulating phase due to a nesting process. Indeed, because $x-2 y \approx 0$ in this range, the conduction band should be about half filled. In the simplest approximation for the copper $\left(x^{2}-y^{2}\right) \mathrm{d}$ band, where $\mathrm{Cu}-\mathrm{O}-\mathrm{Cu}$ transfers are treated as perturbations, exact nesting conditions for $Q= \pm \frac{\pi}{2 a} \pm$ $\frac{\pi}{2 b}$ are realized in the orthorhombic [28] as well as in the tetragonal phase (Fig. 6a,b). For such nesting wave vectors commensurate with a reciprocal lattice vector, Umklapp terms in electron scattering could become relevant and promote the suppression of charge degrees of freedom with a concomitant

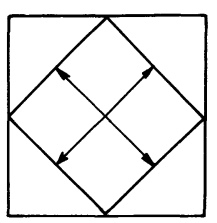

a)

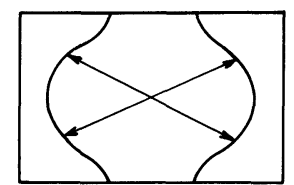

b)
Fig.6.- Nesting conditions for half filled band : a) tetragonal phase ; b) orthorhombic phase. vanishing of superconductivity. This situation is reminiscent of superconductive-insulator transition in organic superconductors of the (TMTTF $)_{2} \mathrm{X}$ series, where a spin density wave or spin Peierls state develop at low temperature even at moderate values of the interchain Coulomb repulsion when $g_{3}$ Umklapp terms become pertinent $[29,30]$. There is indeed some evidence of a SDW state below $240 \mathrm{~K}$ for small values of $x$ in the $\mathrm{La}_{2-x} \mathrm{Sr}_{x} \mathrm{CuO}_{4-y}$ series [19,31]. More work is needed to check whether our samples with $x \approx 0.04 \pm 0.02$ show some evidence of such a phase change.

For $x=0$, the practical absence of Meissner effect in our samples suggests a very inhomogeneous superconductivity, which probably only develops along the grain boundaries with an inhomogeneous repartition of $\mathrm{O}$ vacancies. If the grains are insulating, the preceeding discussion suggests that they are nearly stoichiometric in $\mathrm{O}$, and that $\mathrm{O}$ vacancies concentrate near the grain boundaries. Indeed the weak resistivity anomaly observed by us and others $[18,19]$ at about $240 \mathrm{~K}$ in our undoped samples suggests that the center of the grains has the expected SDW phase. The finding of the same $O$ deficiency in A and B samples by thermogravimetric study, which is a volume determination of $O$ vacancies, supports the picture for a surface only superconductivity of sample A.

If $\mathrm{O}$ vacancies are concentrated, in our undoped samples, near the grain boundaries, this means that the local shift of Fermi level due to $O$ vacancies is larger in the superconductive phase than that due to the average value $y=0.02$. One could thus be locally nearer to a van Hove anomaly of the orthorhombic phase. This would lead to a higher expected $T_{\mathrm{c}}$. However a second effect might then have to be taken into account : if a sizeable fraction of the $\mathrm{O}$ vacancies are in the $\mathrm{CuO}_{2}$ planes, each $\mathrm{O}$ vacancy and its neighbouring $\mathrm{Cu}^{+}$ions provide a strong scattering center for the conductive electrons, which could produce $2 \mathrm{D}$ localization effects on these electrons. This would explain the difference of $\rho(T)$ behaviour of our samples between $x=0$ and $x=0.08$.

Data on annealing superconducting samples under vacuum [32] are in fair agrement with our picture as an increase of $O$ vacancies should shift the Fermi level towards the centre of the band, promote localization and suppress superconductivity.

In conclusion, the purpose of this note was to draw attention on two major experimental evidences (i) the role of oxygen vacancies on the superconductivity phenomenon of $\mathrm{La}_{2} \mathrm{CuO}_{4-y}$ or lightly $\mathrm{Sr}$ substituted samples $(x<0.08)$ and (ii) the role of $\mathrm{Sr}$ substitution on both the $T \rightarrow 0$ transition and the occurrence of a maximum in the superconducting $T_{\mathrm{c}}$.

The observations are coherent with a simple BCS 
scheme and a band filling governed by a balance between $\mathrm{O}$ vacancies and $\mathrm{Sr}$ substitution. Transport properties data suggest that disorder induced localization plays a crucial role on the behaviour of the resistivity above the onset of superconductivity in $\mathrm{La}_{2} \mathrm{CuO}_{3.98}$. We also propose that the band becomes half-filled at a very low concentration of Sr-substitution $(x \approx 0.04)$ leading to a carrier localization via Umklapp scattering in near nested 2D bands with no loss in the spin degrees of freedom some what similar to the observation in $2: 1$ organic conductors. More experiments on varying and controlling the content of $\mathrm{O}$ vacancies, especially in $\mathrm{La}_{2-x} \mathrm{Sr}_{x} \mathrm{CuO}_{4-y}$ with $x$ small or equal to zero, are under progress to check the validity of such a model [33].
Finally, a realistic model should analyse in some details the coupling term leading to the e-e pairing (thus the weak phonon mode associated with the $T \rightarrow 0$ transition near $x_{\mathrm{c}}$ could be partly responsible for the maximum of $T_{\mathrm{c}}$ observed at $x_{\mathrm{c}}$ ) [26].

But the failure to observe any isotope effect on oxygen [34] could suggest e-e coupling through exchange of antiferromagnetic fluctuations [35]. A wealth of experimental and theoretical evidences support the existence of such spin-fluctuation mediated interchain pairing in quasi-1D organic superconductors up to temperatures of about $30-40 \mathrm{~K}$ [36,37].

This work has been partly supported by the DRET contract $86 / 057$. We thank J.C. Ameline and T. Pourthie for their skillful technical help.

\section{References}

[1] Bednorz J.G. and Muller K.A., Z. Phys. B64 (1986) 189.

[2] Foex M., Mancheron A. and Line L. C. R. Heb. Acad. Sci. Paris 250 (1960) 3027.

[3] Longo J. and RACCAH P.M., J. Solid State Chem. 6 (1963) 526.

[4] Shaplygin I.S. KAKHAN B.G. and LAZAREV V.B., Zh. Neorg. Khim. 24 (1979) 1478 [J. Inorg. Chem. 24 (1979) 820].

[5] Michel C. and Raveau B., Rev. Chim. Miner. 21 (1984) 407.

[6] Shamoto S., Hosoya S., Onoda M. and Sato M., submitted to Japan J. Appl. Phys.

[7] TAKagi H., UChida S., KiTAZawa K. and TANAKA S., preprint.

[8] Cava R.J., van Dover R.B., BatlogG B. and Rietman E.A., Phys. Rev. Lett. 58 (1987) 408.

[9] Beille J., Cabanel R., Chaillout C., Chevallier B., Demazeau G. Deslandes F., Etourneau J., Lejay P., Michel C., Provost J., Raveau A., Sulpice A., TholenCe J.L. and Tournier R., C.R. Heb. Acad. Sci. Paris, under press.

[10] Friend R.H., JÉrome D., Frindt R.F., Grant A.J. and Yoffe A.D., J. Phys. C10 (1977) 1013.

[11] Moret R., Pouget J.P. and Collin G., submitted to Europhys. Lett.

[12] van Dover R.B., CAVA R.J., BAtlogg B. and Rietman E.A., Phys. Rev. B35 (1987) 5337.

[13] Cava R.J., Santoro A., Johnson D.W., Jr. and RHODES W.W., preprint.

[14] Nguyen N., Choisnet J., Hervieu M. and Raveau B., Solid State Chem. 39 (1981) 120.

[15] Leblanc, Choisnet J., Bassat J.M. and OdieR $\mathrm{Ph}$., to be published.

[16] Jorgensen J.D., SChuttler H.-B., Hinks D.G., CAPONE Jr. D.W., ZhaNG K., BRODSKY M.B. and Scalapino D.J., Phys. Rev. Lett. 58 (1987) 1024.
[17] SENOUSS S. and MONOD P., private communication. [18] JANOSSY A., private communication to FRIEDEL J.

[19] Greene R.L., Maletta H., Plaskett T.S., BedNORZ J.G. and MULLER K.A., submitted to Solid State Commun.

[20] Baskaran G., Zon Z. and Anderson P.W., Solid State Commun., under press.

[21] Alexandrov A.S., RANNinger J. and RobaskieQICZ S., Phys. Rev. B33 (1986) 4526.

[22] Prelobsek P., Rice T.M. and Zhang F.C., preprint.

[23] Cyrot M., submitted to Solid State Commun.

[24] SChutTler H.D., JoRgenSEN J.D., Hinks D.C., CAPONE Jr. D.W. and SCAlapino D.J., preprint.

[25] LABBE J. and BOK J. Europhys. Lett. 3 (1987) 1225.

[26] BARISIC S., BAtistiĆ I. and Friedel J. Europhys. Lett. 3 (1987) 1231.

[27] Labbe J., Phys. Rev. 172 (1968) 451.

[28] FRIEDEL J., to be published.

[29] Coulon C., Delhaes P., Flandrois S., Lagnier R., Bonjour E. and FABre J.M., J. Physique 43 (1982) 1059.

[30] Emery V.J., Bruinsma R. and Barisic S., Phys. Rev. Lett. 48 (1982) 1039.

[31] Bednorz J.G., TAKashige M. and Müller K.A., Europhys. Lett. 3 (1987) 379.

[32] Shamoto S., Hosoya S., Onada M. and Sato M., Jpn. J. Appl. Phys. 26 (1987) 493.

[33] KANG W. et al., to be published.

[34] Communication of AT\&T at MRS, Conference April, 1987.

[35] EMERY V.J., Synthetic Metals 13 (1986) 21.

[36] Bourbonnais C. and Caron L.G., Phys. Rev. Lett.(1987) to be published.

[37] Jérome D., Creuzet F. and Bourbonnais C., Proceedings of the Yamada Conference $X V$, p. 329, Lake Kawaguchi, Yamanashi, Japan, May 26-30, 1986. 